\title{
Experimental investigation of rotation resistance moment energy spectra in multicylindrical circular Couette system with independently rotating cylinders
}

\author{
Anatoly Serov ${ }^{1,2^{*}}$, Aleksandr Nazarov ${ }^{1,3}$, and Valery Mamonov ${ }^{1}$ \\ ${ }^{1}$ Kutateladze Institute of Thermophysics, Siberian Branch of the RAS, Novosibirsk, Russia \\ ${ }^{2}$ Novosibirsk state technical University, Novosibirsk, Russia \\ ${ }^{3}$ Novosibirsk state University, Novosibirsk, Russia
}

\begin{abstract}
The torque of the rotational resistance in the Ku-Etta multicylinder system rotating in the direction towards each other is measured. The experiments were carried out for three values of the kinematic viscosity of the working fluid that fills the multicylinder system: water at a temperature of $24{ }^{\circ} \mathrm{C}$ (viscosity $0.9 \mathrm{cSt}$ ), an aqueous solution of glycerol at $20^{\circ} \mathrm{C}$ and $41{ }^{\circ} \mathrm{C}(2.5 \mathrm{cSt}$ and $5.2 \mathrm{cSt})$. An attempt is made to investigate the features of a viscous flow in the multicolor Couette flow system from the analysis of the energy spectra of the moment of resistance to rotation of cylinders.
\end{abstract}

\section{Introduction}

Searching for the most cost-effective heat resources for liquid heating led us to the usage of dissipative effects, characterizing an ability of a liquid to interact with solid surfaces. Use of mechanical power of wind rotors with vertical axis for driving liquid heat generators takes special place. In particular, it is of interest to consider a device, where the heat is released in a liquid volume placed in the narrow annular gap between the coaxial rotating towards each other cylinders, as a such heat generator [1,2].

A large number of experimental and theoretical researches has been published concerning the flow between concentric rotating cylinders (circular Couette flow) since the first investigations of Mallock $(1888,1896)$ and Couette (1890) were conducted. The authors [3] demonstrated a detailed map of current regimes in circular gap between rotating towards each other cylinders as Reynolds numbers' functions for inner and outer cylinders $\left(\mathrm{Re}_{i}\right.$ and $\mathrm{Re}_{0}$ respectively) based on proper research. Optical method of polymer flakes distribution in water suspension registration applied by authors [3] made it possible to describe actions and energy spectra of complex structural formations appearing in the circular gap in a wide range of Reynolds numbers' change.

\footnotetext{
${ }^{*}$ Corresponding author: $\underline{\text { serov@itp.nsc.ru }}$
} 


\section{Results}

The article which continues to [2] deals with an attempt to study the features of a viscous liquid flow in a multi-ring Couette system with rotating towards each other coaxial cylinders analyzing rotation resistance moment energy spectra for these cylinders. An experimental setup sceme and a photograph of a main part - heat generator with milticilyndrical rotor system - are shown on the figure 1. Role of the wind rotors in the sceme is performed with low-speed electric motors with variable rotating speed. The setup allows to study the effect of used liquid parameters and «rotor» rotation rate on their resistance to counter-rotation depending on used liquid flow structure in multi-cylindrical «rotor» system gaps. The measurement of total «rotor» rotation resistance moment was conducted as follows: lower drive stator was slowed down, upper drive stator was suspended on a freely rotating suspension axis. Total opposite «rotor» rotation resistance moment was transferred through lever $(L=195 \mathrm{~mm})$ to dynamometer tesometric sensor 4 (figure 1). Dynamometer, thermometer and two-channel tachometer data were transferred through microprocessor data acquisition and pre-processing unit to computer for final processing and storage.

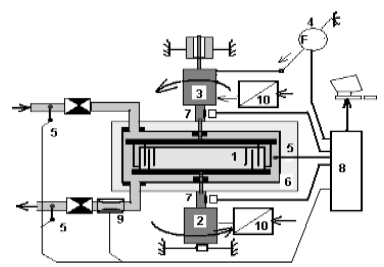

a.

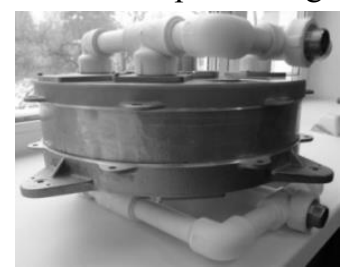

b.

Fig. 1. Scheme of the experimental setup (a) and photograph (b) of the block of the heat source. 1 "the rotors"; 2, 3 - drive; 4 - digital dynamometer; 5 - temperature sensor; 6 - insulation; 7 tachometer; 8 - microprocessor unit for data processing; 9 - flowmeter; 10 - control unit rotation speed of the drive.

Based on measurement of total «rotor» rotation resistance moment the capacity released from heat generator was determined with the formulae: $N=M \Omega$, where $N$ [W] - power, $M$ $[N \cdot m]$ - total moment measured, $\Omega[1 / \mathrm{s}]$ - rotational rate equal to arithmetical mean value of rotational speeds of upper and lower «rotors» towards to fixed coordinate system. Heat power released with heat generator was also determined from these calculations. A comparison of heat generator released power measurement results resulting from measurement of «rotor» rotation resistance moment and from heat measurements is shown on the figure 2. Here $R e=\Omega R \delta / v$ - Reynolds' number for multi-cylindrical system, $R-$ mean system cylinder radius, $\delta$ - mean gap between system cylinders, $v$ - used liquid kinematic viscosity. Power results measured with two independent methods coincide with the high degree of accuracy, which confirms the reliability of the method used for heat generator released power measurement.

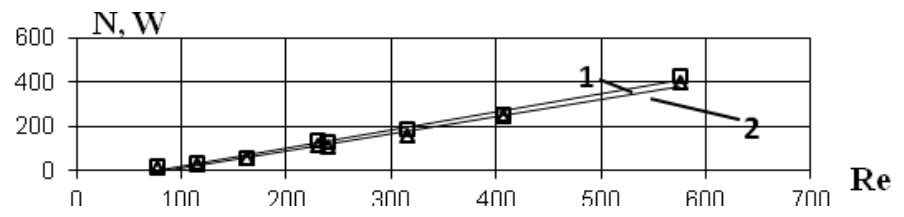

1 - heat power, 2- mechanical power

Fig. 2. Comparison of heat and mechanical power emitted by the heat source. 
The article deals with spectra investigation in the range of small Taylor numbers $(\mathrm{Ta}=$ $\operatorname{Re} \sqrt{\delta / R}) T a=(1-400)$, where the impact of appearing wave vortical flow on rotation resistance is observed, which greatly determines heat processes power in gaps.

There is an area $f_{2}=(0-75) \mathrm{Hz}$ of the main energy with intensity $(\sim 50 \mathrm{~dB})$ and two frequency bands $f_{3,4}=(160-240 ; 235-360) \mathrm{Hz}$ with energy $(\sim 15 \mathrm{~dB})$. It should be noted, that spectral analysis was conducted using ten average spectra for each rotation speed. For more detailed studying these spectra were divided to 5 frequency ranges (figure 3 ).

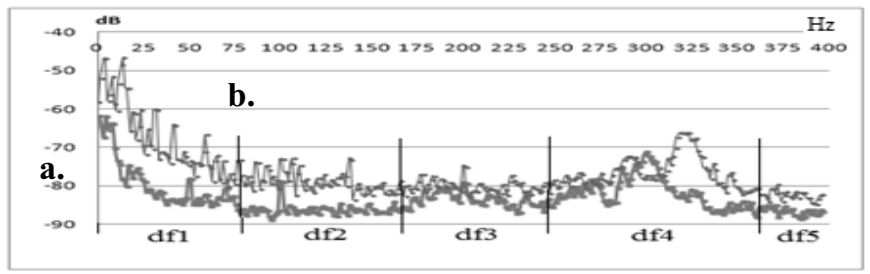

Fig. 3. Mean energy spectra at opposite «rotor» rotation regime for two rotation rates. a $\Omega=15.5$, $1 / \mathrm{s} ; \mathrm{b}-\Omega=28.1,1 / \mathrm{s}$.

Data concerning power input for individual harmonics of the whirl structures to total energy for marked frequency areas are presented in table 1 based on spectral analysis (figure 3). The data show that the main input comes from low-frequency range pulsation.

Table 1. Data power individual harmonics.

\begin{tabular}{|c|c|c|c|}
\hline \multicolumn{2}{|c|}{ Frequency band, Hz } & $\mathbf{\%}$, harmonics, $\mathbf{\Omega}=\mathbf{2 8 . 1}, \mathbf{1 / s}$ & $\mathbf{\%}$, harmonics, $\mathbf{\Omega}=\mathbf{1 5 . 5 , \mathbf { 1 }} \mathbf{s}$ \\
\hline df1 & $0.3-75$ & 95.32 & 68.75 \\
\hline df2 & $75-165$ & 1.01 & 3.77 \\
\hline df3 & $165-240$ & 0.52 & 5.84 \\
\hline df4 & $240-360$ & 3.03 & 20.49 \\
\hline df5 & $360-395$ & 0.14 & 1.28 \\
\hline
\end{tabular}

Subrange $d f 1(0-75 \mathrm{~Hz})$ is different due to the fact that it gives the greatest input to the released heat powery. These data coincide well with the data published in [3] concerning low-frequency pulsation wave spiral appearance (INT) for Reynolds numbers from 20 up to 400. On the figure 4 there are data describing energy input distribution in low-frequency ( 0 $-75 \mathrm{~Hz})$ and in high-frequency spectral areas $(165-355 \mathrm{~Hz})$. The maximum peak capacity values for rotation rate $\Omega=28.11 / \mathrm{s}$ in low-frequency area (figure 4, peaks 1 and 3 ) are 80 times higher than values in high-frequency area (figure 4, peak 9). The peak power values for rotation rate $\Omega=15.51 / \mathrm{s}$ are distributed in the other way: low-frequency values (figure 4 , peaks 5,6 ) are only 10 times higher than similar values in high-frequency area (figure 4 , peaks 7,8$)$.

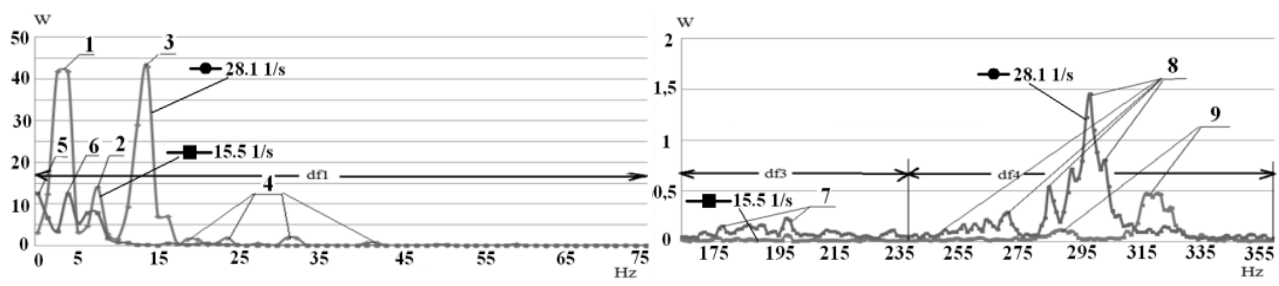

Fig. 4. Frequency power spectrum of the harmonics in the frequency band $F=(0.3-395) \mathrm{Hz}$. Stripe $d f 1(2.7$ - 75) Hz, $d f 3(165-240) \mathrm{Hz}, d f 4(250$ - 360) Hz. 
To obtain more detailed data about whirl formation physical parametres changes in dependance to «rotor» rotation frequency and used liquid viscosity the study of these dependancies must be done while scanning in increments of $d \Omega=11 / \mathrm{s}$ in the rotation rate area studied $\Omega=(5-30) 1 /$ s.

In table 2 there are data presented concerning azimuthal wave numbers for whirl formations $\left(g=f_{\text {harmonics }} / f_{\text {rotation }}\right)$ at rotation rate $\Omega=(15.5$ and 28.1$) 1 / \mathrm{s}$. The data obtained show that spiral and whirl azimuthal size is usually smaller than rotor gap periphery of a circle and slightly depends on rotation rate.

Table 2. Azimuthal wave numbers.

\begin{tabular}{|c|c|c|c|c|}
\hline & $\begin{array}{c}\text { frequency harmonic } \\
\text { (Hz) at } \mathbf{\Omega}=\mathbf{2 8 . 1} \mathbf{1 / \mathbf { s }}\end{array}$ & $\begin{array}{c}\text { frequency harmonic } \\
(\mathbf{H z}) \text { at } \boldsymbol{\Omega}=\mathbf{1 5 . 5}, \mathbf{1 / \mathbf { s }}\end{array}$ & $\begin{array}{c}\mathbf{g} \text { for } \boldsymbol{\Omega}=\mathbf{2 8 . 1}, \\
\mathbf{1 / \mathbf { s }}\end{array}$ & $\begin{array}{c}\mathbf{g} \text { for } \boldsymbol{\Omega}=\mathbf{1 5 . 5}, \\
\mathbf{1 / \mathbf { s }}\end{array}$ \\
\hline f1 & 1.5 & 0.3 & 0.38 & 0.15 \\
\hline f2 & 2.7 & 1.5 & 0.68 & 0.75 \\
\hline f3 & 4 & 4 & 1 & 2 \\
\hline f4 & 7.6 & 6.4 & 1.9 & 3.2 \\
\hline f5 & 11.3 & 7.6 & 2.83 & 3.8 \\
\hline f6 & 12.5 & & 3.13 & \\
\hline f7 & 13.7 & & 3.43 & \\
\hline f8 & 15 & & 3.75 & \\
\hline
\end{tabular}

\section{Conclusion}

- low-frequency spectra $(0-75 \mathrm{~Hz})$ slightly changes in width with rotation rate increase and attributes a lot to resistance moment;

- In the area 4 of the spectra $(165 \mathrm{~Hz}-355 \mathrm{~Hz})$ there are some maximum drifts with rotation rate change, resistance moment inputs in dependance on rotation rate differ insignificantly.

The results of the experiments conducted may serve as a base for the next theoretical and experimental research while creating effective systems of alternative supplies of thermal energy generation.

\section{References}

1. A.F. Serov, V.N. Mamonov, V.I. Terekhov, A.D. Nazarov, Patent for invention "Opposite wind-head generator", № 2612237, 03.03.2017 (2017)

2. V.N. Mamonov, A.D. Nazarov, A.F. Serov, Thermophys. Aeromech. 23, 139 (2016)

3. C.D. Andereck, S.S. Liu, H.L. Swinney, J. Fluid Mech. 164, 155 (1986) 